\title{
Aperfeiçoamento de modelo de estimativa da eficiência de remoção de turbidez da água em floculadores tubulares helicoidais
}

\author{
Model improvement for estimating of water \\ turbidity removal efficiency in helical tubular flocculators
}

Bruno Peterle Vaneli'* (D), Edmilson Costa Teixeira' (1)

$\square$

\begin{abstract}
RESUMO
Estudos científicos têm demonstrado que os floculadores tubulares helicoidais (FTHs) têm alta eficiência na formação de flocos e baixo tempo de retenção hidráulica, quando comparados aos floculadores comumente usados em tratamento de água e esgoto. No entanto, sua aplicação prática é limitada, pois ainda existe demanda significativa por avanços na compreensão da relação entre a hidrodinâmica da unidade e o processo de floculação, bem como critérios e metodologias para auxiliar em projeto racionais de FTH. Nesse contexto, este estudo teve por objetivo propor um aperfeiçoamento no modelo de estimativa de eficiência de remoção de turbidez apresentado por Oliveira (2014), o qual leva em conta um conjunto de parâmetros geométricos, hidráulicos e hidrodinâmicos relevantes ao processo de floculação nesse tipo de reator, pela incorporação de um dos parâmetros mais representativos de processos de floculação, o gradiente de pressão normal (GP ), como uma de suas variáveis independentes. O desenvolvimento do trabalho empregou dinâmica dos fluidos computacional (CFD) no estudo de 84 configurações de FTH, contemplando regimes de escoamento laminar e turbulento. Como resultado, chegou-se a uma nova versão de modelo de estimativa da eficiência de remoção de turbidez da água que, em relação à versão original: tem menor número de variáveis independentes; apresenta melhor ajuste aos dados experimentais; e é mais simples do ponto de vista operacional.
\end{abstract}

Palavras-chave: dinâmica dos fluidos computacional; floculador tubular helicoidal; gradiente de pressão normal; eficiência de floculação; tratamento de água.

\section{ABSTRACT}

Scientific studies have been demonstrating that helical tubular flocculators (HTFs) have high efficiency in floc formation and low hydraulic retention time when compared to flocculators commonly used in water and wastewater treatment. However, its practical application is still limited because there is still a significant demand for advances in the understanding of the relationship between the hydrodynamics of the unit and the flocculation process, as well as for criteria and methodologies in support to the rational design of HTF. In this context, the objective of this study was to propose an improvement in the model of turbidity removal efficiency developed by Oliveira (2014), which takes into account a set of geometric, hydraulic and hydrodynamic parameters relevant to the flocculation process in this type of reactor, by incorporating one of the most representative parameters of flocculation processes, the normal pressure gradient, as one of its independent variables. The development of the work employs computational fluid dynamics (CFD) in the study of $84 \mathrm{HTFs}$ configurations, considering laminar and turbulent flow regimes. As a result, a new model version for estimating water turbidity removal's efficiency in helical tubular flocculators was obtained, which, in relation to the original version, has a smaller number of independent variables, presents better fit to the experimental data and is simpler from the operational point of view. Keywords: computational fluid dynamics; helical tubular flocculators; normal pressure gradient; flocculation efficiency; water treatment.

\section{INTRODUÇÃO}

$\mathrm{Na}$ área da engenharia, os tubos helicoidalmente enrolados têm uma gama de aplicações práticas, tais como trocadores de calor, reatores químicos, sistemas de tubulação, refrigeradores, concentradores espirais, entre outras (YU et al., 2003; CIONCOLINI; SANTINI, 2006). Além dessas

aplicações, alguns autores (GROHMANN; REITER; WIESMANN, 1981; AL-HASHIMI; ASHJYAN, 1989; CARISSIMI; RUBIO, 2005; SILVA, 2007; OLIVEIRA, 2008; entre outros) experimentaram a configuração helicoidal como floculadores hidráulicos - floculadores tubulares helicoidais (FTHs) - aplicados ao tratamento de água. 
A experimentação possibilitou verificar que os FTHs se mostram promissores como alternativa às unidades de processos comumente utilizadas para a floculação, considerando a alta eficiência de floculação, o reduzido tempo de detenção hidráulico e a área requerida para sua implantação. Todavia, a aplicação prática dos FTHs no setor de tratamento de água e esgoto ainda é limitada, pois existe demanda significativa por avanços na compreensão da relação entre a hidrodinâmica da unidade e o processo de floculação, bem como uma busca por critérios e metodologias para auxiliar em seu dimensionamento.

Uma das ferramentas empregadas no dimensionamento das unidades de floculação são as formulações de estimativas da eficiência do processo de tratamento. De acordo com Moruzzi e Oliveira (2013), o modelo mais utilizado para estudar a cinética de floculação é o proposto por Argaman e Kaufman (1970). Marques e Ferreira Filho (2017) ressaltam que esse modelo foi desenvolvido após ensaios com reatores de fluxo contínuo, considerando que, na unidade de floculação, a agregação e a ruptura dos flocos ocorrem de forma simultânea e atingem um estado de equilíbrio. Segundo Thomas, Judd e Fawcett (1999), a agregação é decorrente dos encontros de partículas desestabilizadas e é dependente da agitação da massa líquida para aumentar a taxa de encontros. Por outro lado, a ruptura dos flocos ocorre por causa das tensões de cisalhamento que podem ser intensificadas por meio da adição de energia ao escoamento e/ou do aumento do tempo de floculação.

Particularmente para os FTHs, a formulação mais recente é a proposta por Oliveira (2014). Diferentemente das formulações usualmente empregadas em floculadores genéricos, como a de Argaman e Kaufman (1970), que relacionam a eficiência de processo com o comportamento dos coeficientes de agregação e ruptura dos flocos e com dois parâmetros hidráulicos - o gradiente médio de velocidade $\left(G_{m}\right)$ e o tempo de detenção hidráulico $\left(\mathrm{T}_{\mathrm{d}}\right)$-, a formulação proposta pela autora é específica para os FTHs e associa, por meio de uma equação de regressão, a eficiência de processo com parâmetros representativos de sua geometria, hidráulica e hidrodinâmica.

Os parâmetros geométricos utilizados na formulação de Oliveira (2014) foram o adimensional, formado pela razão entre os diâmetros de enrolamento (D) e do tubo (d), e o adimensional, formado pela razão entre o passo de enrolamento (p) e o comprimento do floculador (L). Por outro lado, os parâmetros hidráulicos foram o número de Camp $(\mathrm{Ca})$ - produto entre os parâmetros $\mathrm{G}_{\mathrm{m}}$ e $\mathrm{T}_{\mathrm{d}}$, comumente considerados os principais parâmetros hidráulicos a serem avaliados na floculação (BRATBY; MILLER, 1977; BRIDGEMAN; JEFFERSON; PARSONS, 2009) - e o número de Reynolds, apontando por Silva (2007) e Oliveira (2008) como um parâmetro relevante para explicar as variações da eficiência de floculação.

Além de ser uma formulação desenvolvida particularmente para os FTHs e considerar aspectos hidráulicos, o modelo proposto por Oliveira (2014) inovou ao incorporar aspectos hidrodinâmicos na mesma.
Os parâmetros hidrodinâmicos utilizados na formulação foram: o número de Swirl (Sn), que corresponde à razão entre os momentos angular e linear de um elemento de fluido, associado à intensidade do escoamento secundário (ISHAK; JAAFAR, 2004); a energia cinética específica média $\left(\overline{\mathrm{E}_{\mathrm{ce}}}\right)$, que corresponde à média dos valores de energia cinética por unidade de massa observados ao longo de uma linha de fluxo que compõe o escoamento em FTHs e representa, de forma global, a característica cinemática de elementos de fluido que se movimentam nessa linha (OLIVEIRA, 2014); e a helicidade (H), que associa a vorticidade local e a velocidade às quais um elemento de fluido está submetido, indicando localmente a característica rotacional do elemento de fluido em conjunto com sua característica translacional na direção principal do escoamento (MOFFATT; TSINOBER, 1992). Ressalta-se que todos os parâmetros hidrodinâmicos foram obtidos por meio de modelagem fluidodinâmica computacional (CFD).

Apesar do avanço alcançado por Oliveira (2014), percebeu-se que um parâmetro hidrodinâmico comumente citado na literatura para caracterizar o escoamento em tubos curvados não foi considerado na formulação desenvolvida. Esse parâmetro é o gradiente de pressão agindo na direção normal ao escoamento principal — o gradiente de pressão normal ( $\left.\mathrm{GP}_{\mathrm{p}}\right)$.

O GP $\mathrm{p}$ é um dos fatores, juntamente com a força centrífuga, responsáveis pelo surgimento e continuidade do escoamento secundário na seção transversal do tubo - principal particularidade do escoamento em tubos curvados (CIONCOLINI; SANTINI, 2006; KÜHNEN et al., 2015; GHOBADI; MUZYCHKA, 2016).

Portanto, faz-se necessário estudar o efeito do $\mathrm{GP}_{\mathrm{p}}$ sobre a qualidade do ajuste da formulação de estimativa de remoção de turbidez desenvolvida por Oliveira (2014). Um questionamento que surge, por consequência, refere-se à significância dos parâmetros hidrodinâmicos Sn, $\overline{\mathrm{E}_{\mathrm{ce}}}$ e H componentes da formulação de Oliveira (2014) em refletir as variações da eficiência de floculação em FTHs.

Nesse contexto, este trabalho tem o objetivo de aperfeiçoar a formulação matemática de estimativa de eficiência de processo em FTHs desenvolvida por Oliveira (2014), no que se refere às suas variáveis independentes, analisando o impacto da inserção do parâmetro GP e o grau de significância de cada um dos parâmetros hidrodinâmicos, com o auxílio da modelagem fluidodinâmica computacional.

\section{METODOLOGIA}

\section{Caracterização das configurações de floculadores tubulares helicoidais}

Ao todo, foram estudadas 84 configurações de FTHs, divididas em 12 arranjos, conforme Tabela 1. Cada configuração de reator foi composta de um tubo flexível de comprimento $L(\mathrm{~m})$, de parede lisa, seção 
circular constante de diâmetro d (m) e enrolado em torno de um cilindro de diâmetro $\mathrm{D}(\mathrm{m})$. A distância entre as voltas é dada por b $(\mathrm{m}) \mathrm{e}$ $\mathrm{p}(\mathrm{m})$ é o passo de enrolamento.

A direção de enrolamento do FTH adotada foi a horizontal (Figura 1), e a condição de escoamento foi estimada pela vazão (Q) e pelo número de Reynolds ( $\mathrm{Re})$.

Os dados experimentais, medidos em termos de eficiência de remoção de turbidez após sedimentação, foram obtidos nos trabalhos de Silva (2007) e Oliveira (2008). Em seus experimentos, o mecanismo utilizado no processo de coagulação foi a varredura. As concentrações de solução de sulfato de alumínio e hidróxido de sódio adotadas foram $0,25 \mathrm{e}$ $50 \mathrm{mg} / \mathrm{L}$, respectivamente, para $\mathrm{pH}$ de 6,8. O circuito hidráulico foi alimentado por água sintética com turbidez inicial de $50 \mathrm{uT}$, armazenada em um tanque. Após a mistura passar pelo FTH, o efluente adentrou em um tanque de decantação retangular e chicanado. $\mathrm{O}$ decantador tinha volume de 3,76 L, 3 chicanas de 1,5 mm de espessura, 1 compartimento de entrada e 1 vertedor de saída. Ressalta-se que o mesmo decantador foi utilizado em todos os testes, ou seja, foram mantidas suas características geométricas e uma vazão constante no interior da unidade igual a 0,5 L.min. ${ }^{-1}$ (velocidade média de sedimentação de $0,021 \mathrm{~cm} / \mathrm{s}$ ), de maneira que as alterações percebidas na eficiência de remoção de turbidez resultassem apenas de variações nas características dos FTHs. Na saída do decantador, amostras do efluente foram coletadas para medição da turbidez. O turbidímetro utilizado tinha resolução de 0,01 uT e exatidão de $|2 \%|$. A turbidez remanescente foi obtida em quintuplicata, para cada uma das configurações estudadas, em que o desvio médio observado foi de $5 \%$.

Salienta-se que nos experimentos de Silva (2007) e Oliveira (2008) a floculação não foi avaliada de forma restrita, visto que sua eficiência foi medida indiretamente por meio da turbidez após a sedimentação.
A medição da turbidez em si, devido ao seu princípio de medição, tem limitações para representação do comportamento e dinâmica das partículas no processo. Além disso, a etapa intermediária de sedimentação incorpora ruídos na avaliação do desempenho da floculação.

\section{Aspectos da modelagem dinâmica dos fluidos computacional}

Para aquisição dos valores dos parâmetros hidrodinâmicos $\mathrm{Sn}, \overline{\mathrm{E}_{\mathrm{ce}}}$, $H$, GPp e do parâmetro $G_{m}$, a fim de relacioná-los com a eficiência de remoção de turbidez, utilizou-se de modelagem fluidodinâmica computacional. As simulações foram realizadas no aplicativo computacional $\mathrm{CFX}^{\circledR}$, versão 14.5 , empregando um computador com processador Intel ${ }^{\circledast}$ Xeon $^{\circledast}$ E5-2650 de 8 núcleos (16 threads), memória de 32 GB e HD de 1 TB.

No âmbito da modelagem tridimensional, adotaram-se as seguintes simplificações para o escoamento: regime permanente, isotérmico, incompressível e monofásico (água). Para os FTHs cujo Re na entrada era maior que o número de Reynolds crítico $\left(\mathrm{Re}_{\mathrm{c}}\right)$ empregou-se o modelo

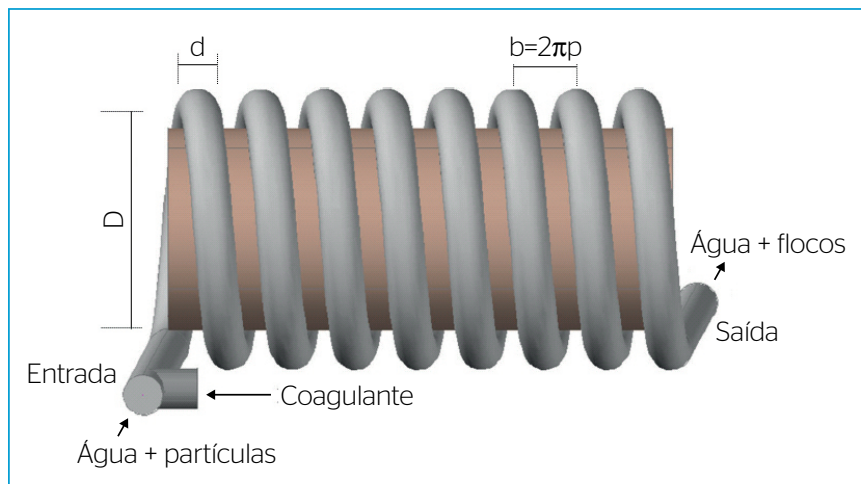

Figura 1 - llustração de uma configuração de floculador tubular helicoidal.

Tabela 1 - Características das configurações de floculadores tubulares helicoidais estudadas.

\begin{tabular}{l|c|c|c|c|c|c|c|c}
\hline Arranjo & Configuração & $\mathrm{d}(\mathrm{m})$ & $\mathrm{D}(\mathrm{m})$ & $\mathrm{D} / \mathrm{d}$ & $\mathrm{p}(\mathrm{m})$ & $\mathrm{L}(\mathrm{m})$ & $\mathrm{Re}$ & $\mathrm{Q}\left(10^{3} \mathrm{~L} . \mathrm{s}^{-1}\right)$ \\
\hline 1 & 1 a 8 & 0,0095 & 0,1135 & 11,92 & 0,0022 & $2,63-36,84$ & 2.496 & 16,7 \\
\hline 2 & 9 a 16 & 0,0095 & 0,1135 & 11,92 & 0,0022 & $2,63-36,84$ & 4.991 & 33,3 \\
\hline 3 & 17 a 24 & 0,0127 & 0,1167 & 9,19 & 0,0027 & $2,96-23,68$ & 1.872 & 16,7 \\
\hline 4 & 25 a 32 & 0,0127 & 0,1167 & 9,19 & 0,0027 & $2,96-23,68$ & 3.744 & 33,3 \\
\hline 5 & 33 a 40 & 0,0159 & 0,1199 & 7,55 & 0,0032 & $1,89-15,16$ & 2.995 & 33,3 \\
\hline 6 & 41 a 48 & 0,0159 & 0,1199 & 7,55 & 0,0032 & $1,89-15,16$ & 5.990 & 66,7 \\
\hline 7 & 49 a 54 & 0,0095 & 0,0635 & 6,67 & 0,0022 & 21,07 & $749-12.479$ & $5,0-83,3$ \\
\hline 8 & 55 a 60 & 0,0095 & 0,3175 & 33,34 & 0,0028 & 21,07 & $749-12.479$ & $5,0-83,3$ \\
\hline 9 & 61 a 66 & 0,0127 & 0,0707 & 5,57 & 0,0033 & 11,84 & $562-9.359$ & $5,0-83,3$ \\
\hline 10 & 67 a 72 & 0,0127 & 0,3167 & 24,94 & 0,0027 & 11,84 & $562-9.359$ & $5,0-83,3$ \\
\hline 11 & 73 a 78 & 0,0127 & 0,3167 & 24,94 & 0,0080 & 11,84 & $562-9.359$ & $5,0-83,3$ \\
\hline 12 & 79 a 84 & 0,0127 & 0,3167 & 24,94 & 0,0159 & 11,84 & $562-9.359$ & $5,0-83,3$ \\
\hline
\end{tabular}


de turbulência k- $\omega$ na modelagem CFD. Os valores do $\mathrm{Re}_{\mathrm{c}}$ foram obtidos de acordo com a Tabela 2, conforme orientado em Vaneli (2016).

Foram empregadas como governantes as Equações 1 e 2 para o modelo laminar e as Equações 3 e 4 para o modelo de turbulência k- $\omega$ padrão.

$\frac{\partial \mathrm{U}_{\mathrm{i}}}{\partial \mathrm{x}_{\mathrm{i}}}=0$

$\rho\left(\mathrm{U}_{\mathrm{j}} \frac{\partial \mathrm{U}_{\mathrm{i}}}{\partial \mathrm{x}_{\mathrm{j}}}\right)=-\frac{\partial \mathrm{P}}{\partial \mathrm{x}_{\mathrm{i}}}+\mu \frac{\partial \mathrm{P}}{\partial \mathrm{x}_{\mathrm{i}}}\left[\left(\frac{\partial \mathrm{U}_{\mathrm{i}}}{\partial \mathrm{x}_{\mathrm{j}}}-\frac{\partial \mathrm{U}_{\mathrm{j}}}{\partial \mathrm{x}_{\mathrm{i}}}\right)\right]+\rho \mathrm{g}_{\mathrm{i}}$

$\frac{\partial \mathrm{k}}{\partial \mathrm{t}}+\overline{\mathrm{U}}_{\mathrm{i}} \frac{\partial \mathrm{k}}{\partial \mathrm{x}_{\mathrm{i}}}=\tau_{\mathrm{ij}} \frac{\partial \overline{\mathrm{U}}_{\mathrm{i}}}{\partial \mathrm{x}_{\mathrm{j}}}-\beta^{\star} \mathrm{k} \omega+\frac{\partial}{\partial \mathrm{x}_{\mathrm{j}}}\left[\left(\vartheta+\vartheta_{\mathrm{t}} \sigma_{\mathrm{k}}\right) \frac{\partial \mathrm{k}}{\partial \mathrm{x}_{\mathrm{j}}}\right]$

$\frac{\partial \omega}{\partial t}+\bar{U}_{i} \frac{\partial \omega}{\partial x_{i}}=\alpha \frac{\omega}{k} \tau_{i j} \frac{\partial \bar{U}_{i}}{\partial x_{j}}-\beta \omega^{2}+\frac{\partial}{\partial x_{j}}\left[\left(\vartheta+\vartheta_{t} \sigma_{\omega}\right) \frac{\partial \omega}{\partial x_{j}}\right]$

Em que:

$\mathrm{U}_{\mathrm{i}, \mathrm{j}, \mathrm{k}}\left(\mathrm{m} \cdot \mathrm{s}^{-1}\right)=\mathrm{a}$ componente da velocidade instantânea nas direções $\mathrm{x}_{\mathrm{i}, \mathrm{j}, \mathrm{k}}$; $\mathrm{P}(\mathrm{Pa})=\mathrm{a}$ pressão estática instantânea;

$\mathrm{g}_{\mathrm{i}}\left(\mathrm{m} \cdot \mathrm{s}^{-2}\right)=$ a componente da aceleração da gravidade na direção $\mathrm{x}_{\mathrm{i}} ;$

$\mathrm{k}=\mathrm{a}$ energia cinética turbulenta $\left(\mathrm{m}^{2} \cdot \mathrm{s}^{-2}\right)$;

$\omega\left(\mathrm{s}^{-1}\right)=$ a taxa de dissipação específica da energia cinética turbulenta.

As constantes do modelo $k-\omega$ padrão são $\beta^{*}=0,09, \alpha=5 / 9, \beta=0,075$, $\sigma_{\mathrm{k}}=2$ e $\sigma_{\omega}=2$.

Como condições de contorno, adotou-se vazão mássica uniforme e normal à entrada. Na saída, foi empregada pressão estática relativa igual a zero (a pressão de referência do domínio foi de $1 \mathrm{~atm}$ ). Nos contornos sólidos aplicou-se condição de não deslizamento, impermeabilidade e paredes lisas. Para os parâmetros de turbulência, especificaram-se os valores dos parâmetros k e $\omega$ na entrada, conforme Equações 5 e 6 , respectivamente. Para a solução do escoamento próximo aos contornos sólidos, adotou-se função de parede automática, disponível no pacote CFX.

$\mathrm{k}=\frac{3}{2} \cdot(\mathrm{U} \cdot \mathrm{I})^{2}$

Tabela 2 - Formulações de estimativas dos valores do número de Reynolds crítico em tubos helicoidalmente enrolados.

\begin{tabular}{l|c|c}
\hline Faixa & Formulação & Autor \\
\hline $\mathrm{D} / \mathrm{d} \leq 35,7$ & $\operatorname{Re}_{c}=77,2\left(\frac{\mathrm{D}}{\mathrm{d}}\right)+2438$ & Kühnen et al. (2015) \\
\hline $35,7<\mathrm{D} / \mathrm{d} \leq 110$ & $\operatorname{Re}_{c}=12500 /\left(\frac{\mathrm{D}}{\mathrm{d}}\right)^{0.31}$ & Cioncolini e Santini (2006) \\
\hline
\end{tabular}

$\omega=\frac{0,09^{-0,25} \cdot \sqrt{\mathrm{k}}}{l_{c}}$

Em que,

$\mathrm{U}=$ velocidade média do escoamento $(\mathrm{m} / \mathrm{s})$;

$\mathrm{I}=$ intensidade de turbulência, $\mathrm{I}=0,16 .(\mathrm{Re})^{-1 / 8}$; e

$l_{c}=$ escala de comprimento da turbulência $(m), l_{c}=0,038 . d$.

Para o modelo Laminar, gerou-se uma malha que tem características semelhantes à malha adotada por Oliveira (2014) ao estudar as mesmas configurações de FTHs avaliadas no presente estudo. A malha gerada foi formada por 10 camadas de elementos prismáticos próximos à parede do tubo, correspondendo a $20 \%$ do diâmetro da seção transversal, e elementos com tamanho máximo de $10 \%$ em relação ao referido diâmetro.

Por outro lado, para o modelo k- $\omega$ padrão, foi necessário realizar análise da independência dos resultados da simulação em relação ao refinamento da malha numérica - teste de malha.

Para isso, utilizou-se de uma configuração de tubo helicoidalmente enrolado estudado por Webster e Humphrey (1993) d=0,0381 m; $\mathrm{D}=0,693 \mathrm{~m} ; \mathrm{Re}=10500)$. No teste de malha, adotou-se um número de 18 camadas de elementos prismáticos próximos à parede do tubo. A espessura da primeira camada foi de 6,3788E-05 m em relação à parede e a taxa de crescimento da espessura das camadas seguintes foi de 1,2 .

Destaca-se que as 18 camadas de prismas corresponderam a 20\% do diâmetro da seção transversal. Mantendo esse percentual fixo, variou-se o tamanho máximo do elemento computacional de 15 a 7\% do diâmetro do tubo, resultando em 6 malhas distintas, conforme Tabela 3.

O procedimento metodológico adotado no teste de malha foi semelhante ao empregado por Sartori et al. (2015). Esse procedimento consiste na comparação de valores de determinado parâmetro físico, neste caso velocidade axial e taxa de dissipação específica de energia cinética turbulenta, estimados por cada malha (malhas 1, 2, 3, 4 e 5), com os valores estimados pela malha mais refinada (malha 6). Para a

Tabela 3 - Características das malhas empregadas no teste.

\begin{tabular}{|c|c|c|c|c|}
\hline M & $\begin{array}{l}\text { Tamanho do } \\
\text { elemento }(\mathrm{m})\end{array}$ & $\begin{array}{l}N^{\circ} \text { de } \\
\text { camadas }\end{array}$ & N. de nós & $\begin{array}{c}\text { Tempo de } \\
\text { processamento }\end{array}$ \\
\hline 1 & $0,005715(15 \%$ d) & 18 & 883.328 & $0,12 . T_{\text {ref }}$ \\
\hline 2 & $0,004572(12 \% d)$ & 18 & 1.457 .924 & $0,27 . T_{\text {ref }}$ \\
\hline 3 & $0,00381(10 \%$ d) & 18 & 2.045 .142 & $0,40 \cdot T_{\text {ref }}$ \\
\hline 4 & $0,003429(9 \%$ d) & 18 & 2.504 .673 & $0,55 \cdot T_{\text {ref }}$ \\
\hline 5 & $0,003048(8 \% d)$ & 18 & 3.262 .317 & $0,70 \cdot T_{\text {ref }}$ \\
\hline 6 & $0,002667(7 \% d)$ & 18 & 4.156 .665 & $\mathrm{~T}_{\text {reff }}{ }^{*}$ \\
\hline
\end{tabular}

*Tempo de processamento de 9,30E+04 segundos. 
quantificação dos erros produzidos nesta comparação, utilizou-se o erro quadrático médio normalizado (EQMN), conforme Equação 7.

EQMN $=\sqrt{\frac{1}{n} \sum_{\mathrm{i}=1}^{\mathrm{n}} \frac{\left(\mathrm{m}_{\mathrm{ref}_{\mathrm{i}}}-\mathrm{m}_{\mathrm{i}}\right)^{2}}{\mathrm{~m}_{\mathrm{ref}_{\mathrm{i}}}^{2}}}$

Na qual:

$\mathrm{m}_{\mathrm{i}}=$ valor do parâmetro no ponto $\mathrm{i}$;

$\mathrm{m}_{\text {ref }_{\mathrm{i}}}=$ valor do parâmetro da malha mais refinada no ponto i; e

$\mathrm{n}=$ número de pontos avaliados.

Definida a malha, realizou-se a validação do modelo fluidodinâmico computacional. Para isso foram utilizados dados experimentais do campo de velocidade axial obtidos por Yu et al. (2003) e Webster e Humphrey (1993), para validação do modelo Laminar e do k- $\omega$ padrão, respectivamente. Para a comparação entre o valor medido $\left(\mathrm{m}_{\mathrm{ref}_{\mathrm{i}}}\right)$ e o estimado $\left(\mathrm{m}_{\mathrm{i}}\right)$, empregou-se o EQMN (Equação 7). Ressalta-se que o modelo de turbulência k- $\omega$ foi previamente calibrado. Em todas as simulações utilizou-se o coeficiente $\beta^{*}$ (ver Equação 3 ), coeficiente de calibração, igual a 0,1035 , aumento de $15 \%$ em relação do valor default.

\section{Estimativa dos valores dos parâmetros hidrodinâmicos}

Para a aquisição dos valores dos parâmetros hidrodinâmicos, utilizou-se procedimento metodológico semelhante ao empregado por Oliveira (2014). Os parâmetros $\mathrm{G}_{\mathrm{m}}$, GP, Sn e H foram obtidos em uma seção transversal do escoamento, após o comprimento de entrada, correspondente à quarta volta da configuração de FTH avaliada. Por outro lado, o valor da $\overline{\mathrm{E}_{\text {ce }}}$ foi obtido a partir de uma linha de fluxo do escoamento que partiu do centro da seção transversal após o comprimento de entrada. Os valores do $G_{m}$ e do GP foram adquiridos a partir dos valores pontuais verificados na seção transversal de avaliação (quarta volta), conforme Equação 8 .

$\mathrm{X}_{\mathrm{m}}=\sqrt{\frac{\mathrm{X}_{\mathrm{pl}}^{2}+\mathrm{X}_{\mathrm{p} 2}^{2}+\ldots+\mathrm{X}_{\mathrm{pn}}^{2}}{\mathrm{n}}}$

Em que:

$\mathrm{n}$ = número de elementos discretos na seção transversal avaliada;

$\mathrm{X}_{\mathrm{p}}=$ valor pontual; $\mathrm{e}$

$\mathrm{X}_{\mathrm{m}}=$ médio do parâmetro na seção transversal.

Os valores pontuais do gradiente de velocidade $\left(G_{p}\right)$ foram estimados por meio da Equação 9, conforme Kramer e Clark (1997).

$G_{p}=\sqrt{\left[\left(\frac{\partial u}{\partial x}\right)^{2}+\left(\frac{\partial v}{\partial y}\right)^{2}+\left(\frac{\partial w}{\partial z}\right)^{2}\right]+\left(\frac{\partial u}{\partial y}+\frac{\partial v}{\partial x}\right)^{2}+\left(\frac{\partial u}{\partial z}+\frac{\partial w}{\partial x}\right)^{2}+\left(\frac{\partial v}{\partial z}+\frac{\partial w}{\partial y}\right)^{2}}$
Na qual:

$\mathrm{u}, \mathrm{v}$ e $\mathrm{w}\left(\mathrm{m} \cdot \mathrm{s}^{-1}\right)=$ as componentes da velocidade; $\mathrm{e}$

$\mathrm{x}=$ normal, $\mathrm{y}=$ tangencial $\mathrm{e} \mathrm{z}=$ binormal ao escoamento principal.

Por outro lado, estimaram-se os valores pontuais do $\mathrm{GP}_{\mathrm{p}}$ por meio da variação da pressão total do fluido $\mathrm{P}\left(\mathrm{N} . \mathrm{m}^{-2}\right)$ na direção normal ao escoamento (direção ao longo do eixo x) (Equação 10).

$\mathrm{GP}_{\mathrm{p}}=\frac{\partial \mathrm{P}}{\partial \mathrm{x}}$

Para a estimativa do valor do Sn, foi empregada a Equação 11. Essa equação é uma adaptação realizada por Oliveira (2014) com base em Chigier e Beer (1964) para escoamentos com linhas de fluxo de formato helicoidal.

$\operatorname{Sn}=\frac{\rho \int_{\mathrm{Sc}}\left(\overrightarrow{\mathrm{r}} \times \overrightarrow{\mathrm{V}}_{\text {secundário }}\right) \overrightarrow{\mathrm{v}} \mathrm{d} \overrightarrow{\mathrm{A}}}{\text { r. } \rho \int_{\mathrm{Sc}} \overrightarrow{\mathrm{V}} \overrightarrow{\mathrm{V}} \mathrm{d} \overrightarrow{\mathrm{A}}}$

Na qual:

$\overrightarrow{\mathrm{r}}=$ vetor posição (em relação aos vórtices de rotação formados na seção transversal);

$\overrightarrow{\mathrm{V}}_{\text {secundário }}=$ vetor formado pelos vetores normal e binormal de velocidade; $\mathrm{r}=$ raio do tubo; $\mathrm{e}$

$\mathrm{d} \overrightarrow{\mathrm{A}}=$ elemento de área.

A H do escoamento foi calculada por meio da Equação 12, conforme Moffatt e Tsinober (1992).

$\mathrm{H}=\int_{\forall}(\overrightarrow{\mathrm{V}} \cdot \overrightarrow{\mathrm{W}}) \mathrm{d} \mathrm{V}$

Em que:

$\overrightarrow{\mathrm{W}}=$ vetor vorticidade;

$\overrightarrow{\mathrm{V}}=$ vetor velocidade; $\mathrm{e}$

$\mathrm{d} \mathrm{V}=$ elemento de volume

Por fim, para a aquisição da $\overline{\mathrm{E}_{\mathrm{ce}}}$, empregou-se a Equação 13, proposta por Oliveira (2014).

$\overline{\mathrm{E}_{\mathrm{ce}}}=\frac{1}{\mathrm{n}} \cdot \sum_{\mathrm{i}=1}^{\mathrm{n}} \frac{\mathrm{v}^{2}}{2}$

Na qual:

$n=$ número de elementos discretos ao longo da linha de fluxo avaliada; $\mathrm{e}$ $v=$ magnitude da velocidade na direção tangencial.

É necessário frisar que os parâmetros geométricos, hidráulicos e hidrodinâmicos representativos dos FTHs foram considerados por meio de parâmetros adimensionais na formulação matemática de estimativa de eficiência de remoção de turbidez. 
Para a concepção da formulação de estimativa de remoção de turbidez, correlacionaram-se esses parâmetros adimensionais (variáveis independentes) com a eficiência de remoção de turbidez (variável dependente), por meio de regressão linear.

$\mathrm{Na}$ Tabela 4 são destacados os parâmetros e seus adimensionais correspondentes. Em que $\mathrm{H}_{\mathrm{adm}}, \mathrm{E}_{\mathrm{adm}}$ e $\mathrm{GP}_{\mathrm{adm}}$ são as formas adimensionais adotadas para os parâmetros hidrodinâmicos $\mathrm{H}, \overline{\mathrm{E}_{c e}}$ e gradiente médio de pressão normal, nesta ordem.

\section{RESULTADOS E DISCUSSÃO}

\section{Teste de malha e validação da modelagem dinâmica dos fluidos computacional}

Na Figura 2 é apresentado o resultado do teste de malha com o modelo k- $\omega$. Observa-se que, para ambos os parâmetros normalizados, a curva de EQMN (Equação 7) apresenta comportamento assintótico a partir da malha 3. Para o parâmetro velocidade axial, o valor do EQMN para a malha 3 foi da ordem de $0,85 \%$ para os perfis normal e binormal da seção transversal do tubo. Enquanto para o parâmetro de turbulência, os valores de EQMN obtidos foram de 3,2 e 2,3\% para os perfis normal e binormal, respectivamente. Devido a esse resultado, a malha 3 foi escolhida para aplicação nas simulações cuja modelagem necessitou do emprego do modelo de turbulência.

Na Tabela 5 são apresentados os valores dos parâmetros das malhas utilizadas nas simulações CFD, para o modelo Laminar e k- $\omega$.

Tabela 4 - Parâmetros e formas utilizadas na análise de regressão linear.

\begin{tabular}{lc|c|c|c|c|c|c|c} 
Parâmetro & $\mathrm{D} / \mathrm{d}$ & $\mathrm{p} / \mathrm{L}$ & $\mathrm{Ca}$ & $\mathrm{Re}$ & $\mathrm{Sn}$ & $\mathrm{H}_{\mathrm{adm}}$ & $\mathrm{E}_{\mathrm{adm}}$ & $\mathrm{GP}_{\mathrm{adm}}$ \\
\hline Forma & $\mathrm{D} / \mathrm{d}$ & $\mathrm{p} / \mathrm{L}$ & $\mathrm{G}_{\mathrm{m}} \mathrm{T}_{\mathrm{d}}$ & $\mathrm{p} . \mathrm{d} . \mathrm{U} / \mu$ & - & $\mathrm{H} . \mathrm{T}_{\mathrm{d}} / \mathrm{U}$ & $\overline{\mathrm{E}_{\mathrm{ce}}} / \mathrm{Q} . \mu$ & $\mathrm{GP} . \mathrm{L} / \mathrm{\rho} \mathrm{U}^{2}$
\end{tabular}

Na Figura 3, nota-se, próximo às paredes, o refinamento diferenciado das malhas empregadas nas simulações. Próximo ao contorno sólido, a malha é composta de elementos prismáticos; na região central da seção transversal, a malha é composta por elementos tetraédricos.

O resultado da validação dos modelos Laminar e k- $\omega$ é apresentado nas Figuras 4 e 5, nessa ordem. Os valores do EQMN produzidos pelo modelo Laminar ao estimar os dados experimentais de velocidade ao longo do eixo normal ao escoamento (Figura 4A) e binormal ao escoamento (Figura 4B) foram de 6,6 e 4,8\%, respectivamente, próximos da incerteza máxima da medida, da ordem de 6\%. Esses resultados corroboram Sartori et al. (2015) ao estudarem o mesmo experimento.

No caso do modelo k- $\omega$, os respectivos valores de EQMN verificados na estimativa dos perfis de velocidade axial ao longo do eixo normal para $\mathrm{Re}=3800$ (Figura 5A) e $\mathrm{Re}=10500$ (Figura 5B) foram de 7,8 e 7,2\%. Webster e Humphrey (1993) destacaram que, para as medições realizadas próximas ao contorno sólido, a incerteza experimental era significantemente maior que $3 \%$. Nesse contexto, verificou-se que tanto o modelo Laminar como o modelo k- $\omega$ representaram, conforme suas limitações, a realidade física do tipo de escoamento estudado.

\section{Formulação proposta para estimativa de remoção de turbidez}

Na Tabela 6 são apresentadas as estatísticas globais produzidas pela formulação desenvolvida por Oliveira (2014) e aquela proposta neste

Tabela 5 - Valores dos parâmetros das malhas usadas nas simulações dinâmica dos fluidos computacional.

\begin{tabular}{l|c|c|c} 
Modelos & $\begin{array}{c}\text { Espessura da } \\
\text { camada de prismas }\end{array}$ & $\begin{array}{c}\text { Número de } \\
\text { camadas de primas }\end{array}$ & $\begin{array}{c}\text { Tamanho } \\
\text { do elemento }\end{array}$ \\
\hline Laminar & $0,2 \mathrm{~d}$ & 10 & $0,1 \mathrm{~d}$ \\
\hline $\mathrm{k}-\omega$ & $\mathrm{O}, 2 \mathrm{~d}$ & 18 & $0,1 \mathrm{~d}$ \\
\hline
\end{tabular}

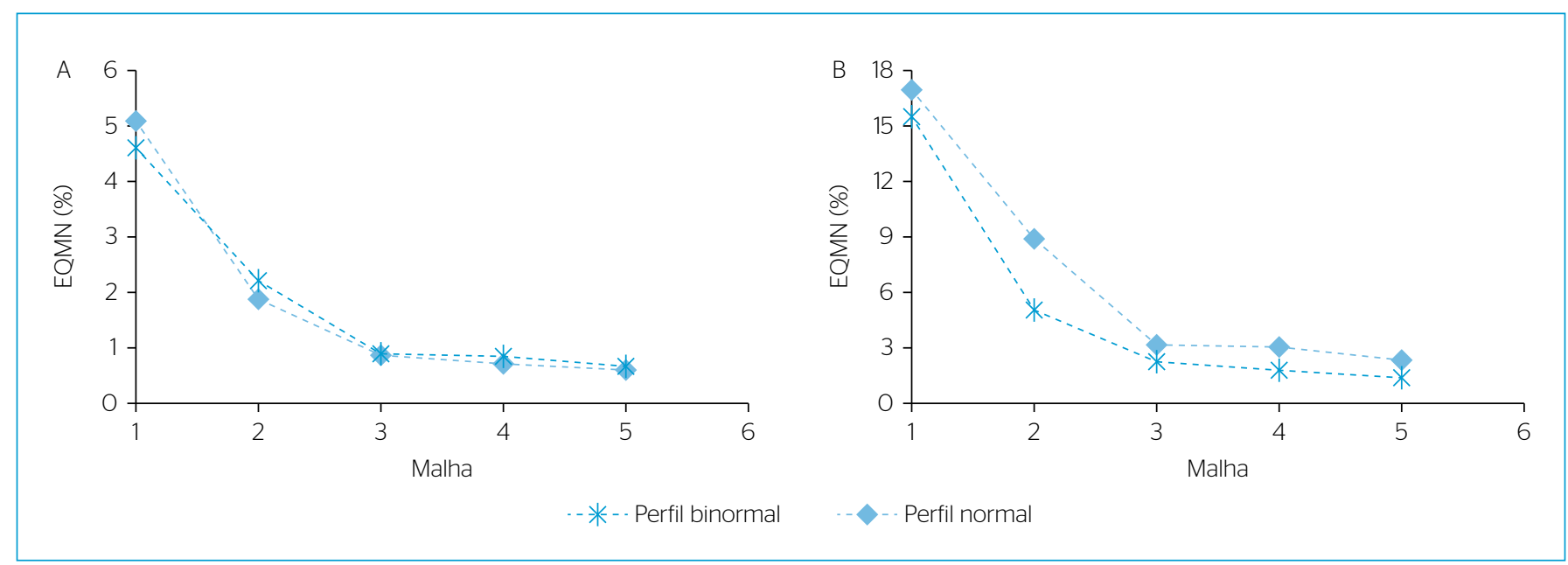

Figura 2 - Erro quadrático médio normalizado resultante da comparação de cada malha com a malha de referência - malha 6. (A) velocidade axia normalizada; (B) taxa de dissipação específica de energia cinética turbulenta normalizada. 


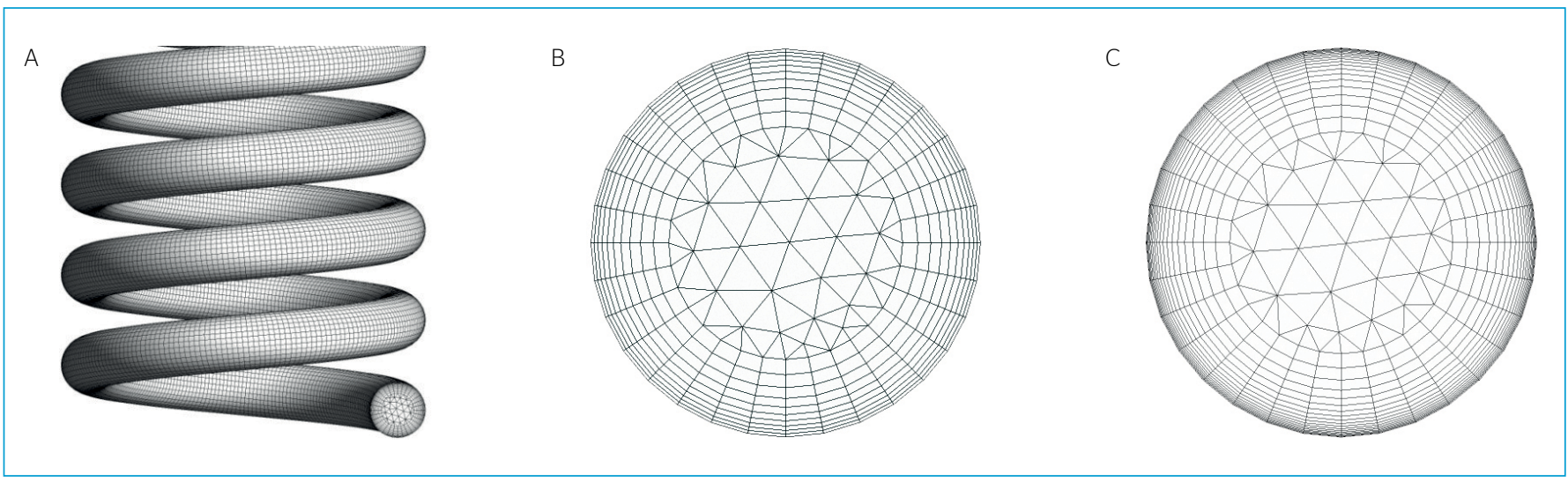

Figura 3 - (A) Ilustração de malha computacional utilizada nas simulações e detalhe do refinamento na região próxima à parede (B) modelo Laminar; (C) modelo k- $\omega$.

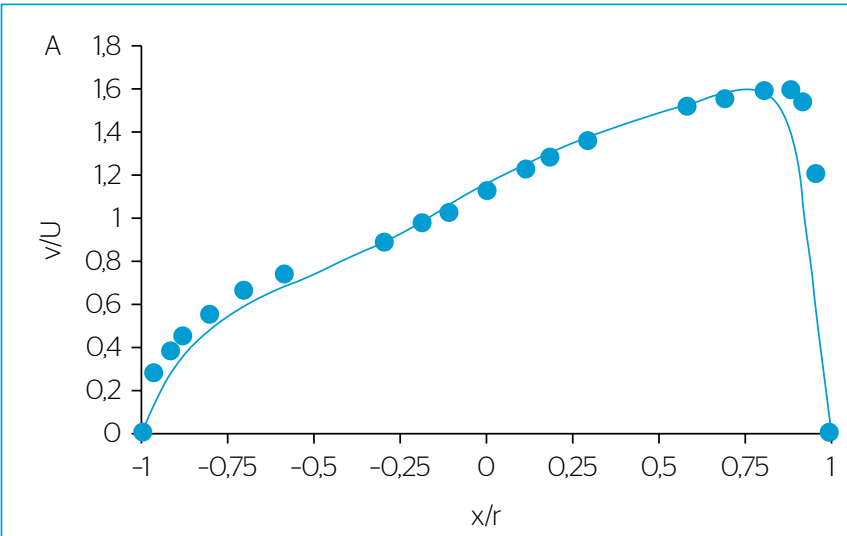

- Experimental

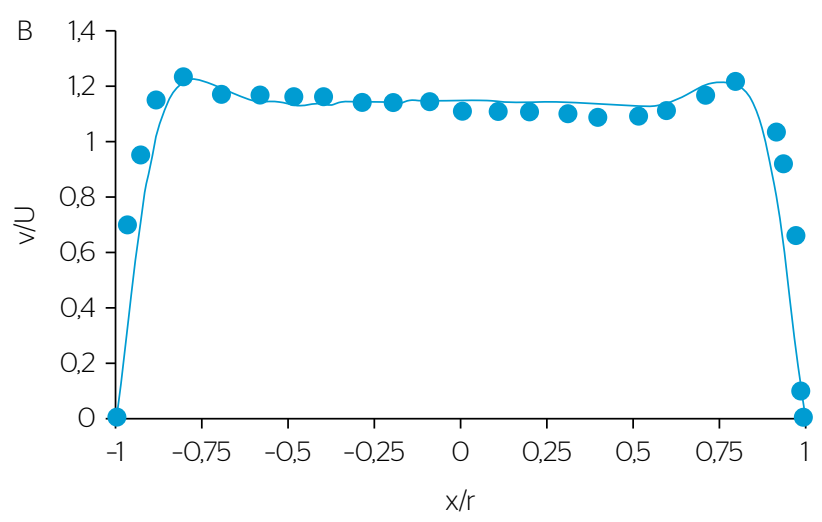

Numérico

Figura 4 - Estimativa do perfil de velocidade axial ao longo do eixo normal (a) e binormal (b) ao escoamento principal pelo modelo Laminar. Parâmetros geométricos (YU et al., 2003): r=0,0047 m; R=0,0637; b=0,0318; Re=2000.

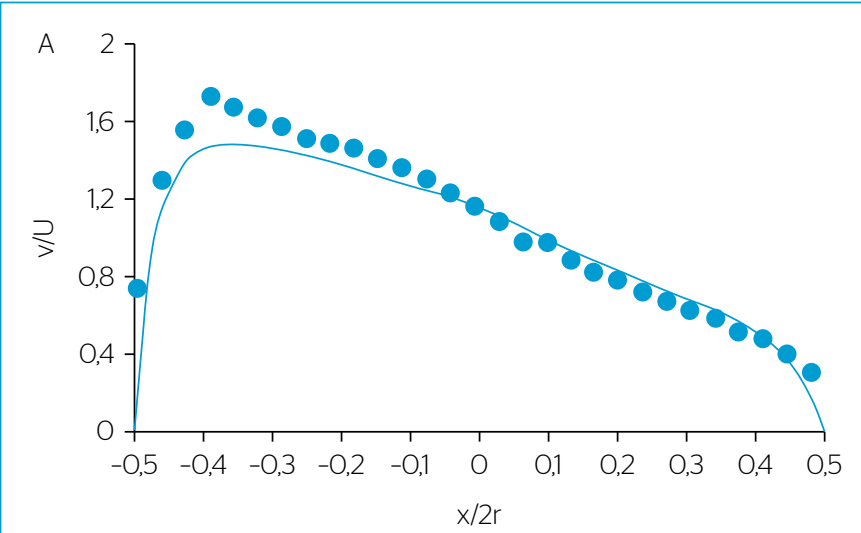

- Experimental

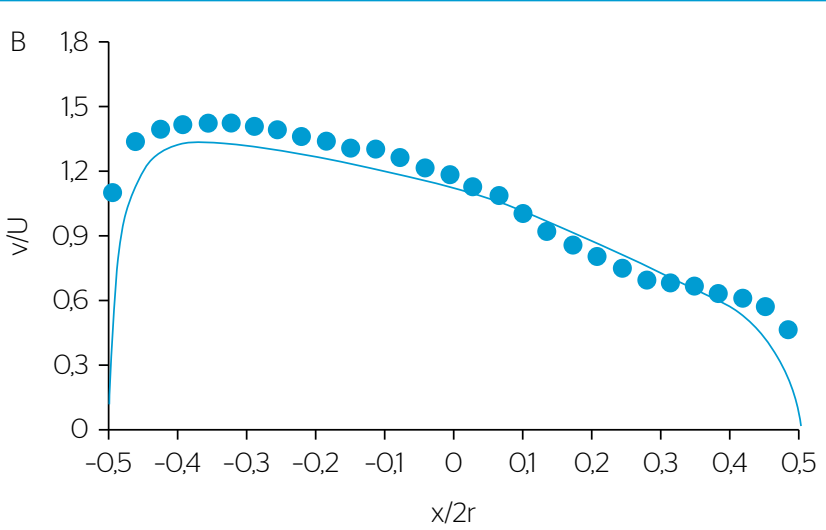

- Numérico

Figura 5 - Estimativa do perfil de velocidade axial ao longo do eixo normal ao escoamento principal pelo modelo $k-\omega$. (A) $R e=3.800$; (B) Re=10.500. Parâmetros geométricos (WEBSTER; HUMPHREY, 1993): r=0,01905 m; R=0,3465 m; b=0,1295. 
trabalho. A diferença principal entre as duas formulações é a presença do parâmetro gradiente médio de pressão adimensional $\left(\mathrm{GP}_{\mathrm{adm}}\right)$ na formulação proposta.

Quanto às estatísticas, observou-se redução da MQR na ordem de $27 \%$ e diminuição do $S_{\mathrm{e}}$ de 2,05 para $1,74 \%$, quando comparadas às duas formulações. Notou-se também aumento no $\mathrm{R}_{\text {ajus }}^{2}$ de 89,04 para $91,86 \%$. Além disso, o valor do $\mathrm{R}_{\text {máx }}$ indicou que a formulação proposta produziu desvios dentro da incerteza experimental da medida da turbidez remanescente (ordem de 5\%).

Dessa forma, a análise conjunta das estatísticas sugeriu que a introdução do $\mathrm{GP}_{\mathrm{adm}}$, como variável independente, gerou uma formulação matemática de estimativa de remoção de turbidez mais aderente ao processo físico-químico, quando comparada à desenvolvida por Oliveira (2014).

Partindo dessa constatação, foi possível realizar uma análise de desempenho da formulação sugerida diante de outras formulações de estimativa da eficiência da floculação comumente utilizadas na literatura, como a desenvolvida por Argaman e Kaufman (1970), citando a avaliação realizada por Oliveira (2014). A autora comparou as estatísticas produzidas por sua formulação e pela formulação de Argaman e Kaufman (1970), aplicando-a às configurações de FTHs correspondentes aos arranjos 1 a 6 (ver Tabela 1). Em sua avaliação, observou que os desvios absolutos máximo e médio produzidos por sua formulação eram uma ordem de grandeza menor que os verificados pela formulação de Argaman e Kaufman (1970).

Quanto ao processo físico da floculação, sabe-se que o $\mathrm{GP}_{\mathrm{p}}$ exerce influência direta sobre a intensidade do escoamento secundário (VANELI, 2016) e que tal gradiente acarreta aumento na intensidade de mistura na seção transversal (AL HASHIMI; ASHJYAN, 1989; CIONCOLINI; SANTINI, 2006), de modo a provocar modificações na distribuição de coloides, partículas em suspensão e até pequenos flocos ao longo das seções do FTH (SARTORI et al., 2015), favorecendo, ao longo de um determinado tempo, a eficiência do processo de floculação.

$\mathrm{Na}$ Tabela 7 são apresentadas as estatísticas associadas aos coeficientes de regressão de cada uma das variáveis independentes, após a inserção do parâmetro $\mathrm{GP}_{\text {adm }}$.

O critério escolhido para inferir acerca da significância das variáveis independentes na formulação de estimativa de remoção de turbidez foi admitir $\mathrm{p}<0,05$. O critério adotado é o nível de significância usualmente empregado como limite.

Tabela 6 - Estatísticas da formulação de Oliveira (2014) e da formulação proposta.

\begin{tabular}{l|c|c|c|c}
\hline Formulação & $\mathbf{S}_{\mathrm{e}}$ & $\mathbf{R}_{\text {máx }}$ & $\mathbf{M Q R}$ & $\mathbf{R}_{\text {ajus }}^{2}$ \\
\hline Oliveira (2014) & $2,05 \%$ & $6,37 \%$ & 0,000419 & $89,04 \%$ \\
\hline Formulação proposta & $1,74 \%$ & $4,06 \%$ & 0,000304 & $91,86 \%$ \\
\hline
\end{tabular}

$\mathrm{S}$ : erro padrão da estimativa; $\mathrm{R}_{\text {ma: }}$ resíduo máximo; $\mathrm{MQR}$ : média dos quadrados dos resíduos; $R_{\text {aus: }}^{2}$ coeficiente de determinação ajustado.
Avaliando a Tabela 7, verificou-se que tanto o parâmetro Sn como o $\mathrm{H}_{\text {adm }}$ sequer atingiram o nível de significância de 0,05 , dando evidência estatística de que existe fraca relação entre eles e a eficiência de remoção de turbidez, nesta formulação. Outro indicativo da fraca relação são os respectivos limites do intervalo de confiança dos coeficientes de regressão, assumindo valores positivos e negativos.

Do ponto de vista do processo físico, interpretar o efeito da $\mathrm{H}$ no processo de floculação é uma tarefa complexa, haja vista que esse parâmetro carrega consigo a informação de dois movimentos combinados, o de rotação local em torno do próprio eixo e o de translação do elemento de fluido na direção do escoamento principal (Equação 12). Analisando os dois movimentos separadamente - uma simplificação - , verifica-se que a translação do elemento de fluido na direção do escoamento principal já é considerada na formulação, por meio do número de Reynolds. Enquanto o efeito do movimento de rotação do elemento de fluido ao redor do seu próprio eixo não contribui para a colisão dos flocos, pois não causa distorção no elemento de fluido (KRAMER; CLARK, 1997).

Quanto ao parâmetro Sn, ele capta o mesmo fenômeno físico que o gradiente médio de pressão normal - o escoamento secundário. Uma possível explicação para o fato de as estatísticas o terem apontado como insignificante na formulação proposta é que o parâmetro $\mathrm{GP}_{\text {adm }}$ pode ser mais sensível para inferir sobre o escoamento secundário do que o Sn. Como apresentado em Vaneli (2016), para uma mesma condição de escoamento, o perfil do gradiente de pressão normal é mais sensível às mudanças na curvatura do tubo do que o perfil de velocidade tomado ao longo do eixo normal da seção de escoamento.

Em termos operacionais, é desejável que as formulações de estimativa de remoção de turbidez representem de modo satisfatório o fenômeno físico-químico da floculação e que sejam o mais simples possível. Neste ponto, destaca-se que, entre todos os parâmetros hidrodinâmicos

Tabela 7 - Estatísticas associadas aos coeficientes de regressão de cada uma das variáveis independentes.

\begin{tabular}{|c|c|c|c|c|}
\hline \multirow{2}{*}{$\begin{array}{l}\text { Variável } \\
\text { Independente }\end{array}$} & \multirow{2}{*}{$\begin{array}{l}\text { Coeficientes } \\
\text { de regressão }\end{array}$} & \multirow[b]{2}{*}{$P$} & \multicolumn{2}{|c|}{ Intervalo de confiança } \\
\hline & & & $\begin{array}{c}95 \% \\
\text { inferiores }\end{array}$ & $\begin{array}{c}95 \% \\
\text { superiores }\end{array}$ \\
\hline Interseção & 7,63E-O1 & 1,04E-47 & 7,19E-01 & 8,07E-01 \\
\hline $\mathrm{Ca}$ & $-3,28 \mathrm{E}-06$ & 7,17E-05 & $-4,83 E-06$ & $-1,73 \mathrm{E}-\mathrm{O} 6$ \\
\hline $\operatorname{Re}$ & $-5,22 \mathrm{E}-06$ & 2,09E-03 & $-8,49 E-06$ & -1,96E-06 \\
\hline$D / d$ & 6,97E-O3 & 4,75E-19 & 5,81E-03 & 8,13E-03 \\
\hline Sn & 9,13E-O1 & $6,88 \mathrm{E}-\mathrm{O} 2$ & $-7,21 \mathrm{E}-\mathrm{O} 2$ & 1,90E+OO \\
\hline$H_{\text {adm }}$ & $-2,49 E-06$ & 3,85E-01 & -8,17E-06 & 3,18E-06 \\
\hline$E_{a d m}$ & -1,07Е-08 & 1,2OE-O4 & $-1,6 \mathrm{OE}-\mathrm{O} 8$ & $-5,46 \mathrm{E}-09$ \\
\hline $\mathrm{p} / \mathrm{L}$ & $-2,92 \mathrm{E}+01$ & 7,55E-06 & $-4,13 E+01$ & $-1,71 \mathrm{E}+01$ \\
\hline $\mathrm{GP}_{\text {adm }}$ & 1,52E-O4 & 5,27E-O4 & 6,86E-05 & 2,36E-O4 \\
\hline
\end{tabular}


citados neste estudo, o Sn foi aquele que ofereceu maior esforço para sua aquisição via modelagem fluidodinâmica computacional.

Dessa forma, a formulação proposta para estimativa da eficiência de remoção de turbidez em FTHs não apresenta os parâmetros Sn e $\mathrm{H}_{\mathrm{adm}}$. A formulação matemática proposta é dada pela Equação 14.

$\mathrm{Ef}=0,800-4,278 \cdot 10^{-6} \mathrm{Ca}-5,909 \cdot 10^{-6} \mathrm{Re}-28,484 \frac{\mathrm{p}}{\mathrm{L}}+$

$6,530.10^{-3} \frac{\mathrm{D}}{\mathrm{d}}-8,351 \cdot 10^{-9} \mathrm{E}_{\mathrm{adm}}+1,824 \cdot 10^{-4} \mathrm{GP}_{\mathrm{adm}}$

Na qual:

Ef = eficiência de remoção de turbidez.

Salienta-se que a formulação apresentada tem natureza empírica, visto que resultou de ajuste linear de parâmetros para as condições específicas do estudo. Além disso, o desempenho da floculação não foi avaliado de forma restrita, pois ele é medido por meio da turbidez após sedimentação.

Na Tabela 8 é apresentada a faixa de variação das variáveis independentes consideradas no desenvolvimento da formulação proposta.

As faixas de variação dos gradientes médios de velocidade e de tempo de detenção empregadas neste trabalho foram 25 a $2550 \mathrm{~s}^{-1} \mathrm{e}$ 6 a 300 s, respectivamente.

$\mathrm{Na}$ Tabela 9 são ilustradas as estatísticas globais produzidas pela formulação desenvolvida por Oliveira (2014) e a proposta neste estudo.

Tabela 8 - Faixa de variação das variáveis independentes consideradas na Equação 14.

\begin{tabular}{l|c|c|c|c|c|c} 
Limites & Ca & Re & $\frac{p}{L}$ & $\frac{D}{d}$ & $E_{a d m}$ & $G_{\text {adm }}$ \\
\hline Inferior & $1,53.10^{3}$ & $5,62.10^{2}$ & $5,84.10^{-5}$ & 5,60 & $1,70.10^{5}$ & 37,80 \\
\hline Superior & $5,53.10^{4}$ & $1,25.10^{4}$ & $1,67.10^{-3}$ & 33,30 & $1,08.10^{7}$ & 931,30 \\
\hline
\end{tabular}

Verificou-se na Tabela 9 que a retirada dos parâmetros $\mathrm{n}_{\mathrm{e}} \mathrm{H}_{\text {adm }}$ produziu alteração insignificante nas estatísticas da formulação proposta e que a formulação final (Equação 14), sem os parâmetros $\mathrm{Sn} \mathrm{e} \mathrm{H}_{\text {adm }}$, apresenta estatísticas melhores que a desenvolvida por Oliveira (2014), apesar de ser mais simples. Destaca-se que: o resíduo máximo produzido pela formulação proposta foi de $4,65 \%$, e se manteve dentro da incerteza experimental da medida (ordem de $5 \%$ ), a soma dos quadrados dos resíduos reduziu de 0,0318 para 0,0240 (redução da ordem de 25\%) e o coeficiente de determinação saltou de 89,96 para $92,28 \%$.

Para verificar a capacidade de predição dos dados experimentais da Equação 14, apresentam-se duas figuras ilustrando comportamentos distintos da eficiência de remoção de turbidez em FTHs. Em ambas figuras, os dados experimentais ilustrados são os mesmos utilizados nos ajustes da formulação proposta e da formulação de Oliveira (2014).

Na Figura 6A são apresentadas as configurações de FTHs correspondentes ao arranjo 2, no qual se observa o comportamento da eficiência de remoção ao longo do tempo de processo.

Por outro lado, na Figura 6B são apresentadas as configurações de FTHs correspondentes ao arranjo 11, no qual se observa o comportamento da eficiência de remoção de turbidez com o aumento do valor do número de Reynolds do escoamento.

Tabela 9 - Estatísticas da formulação de Oliveira (2014) e da formulação proposta.

\begin{tabular}{|c|c|c|c|c|}
\hline Formulação & $\mathrm{S}_{\mathrm{e}}$ & $\mathbf{R}_{\text {máx }}$ & SQR & $\mathbf{R}^{2}$ \\
\hline Oliveira (2014) & $2,05 \%$ & $6,37 \%$ & 0,0318 & $89,96 \%$ \\
\hline Com Sn e $\mathrm{H}_{\mathrm{adm}}$ & $1,74 \%$ & $4,06 \%$ & 0,0228 & $92,64 \%$ \\
\hline Sem Sn e Hadm (Equação 14) & $1,76 \%$ & $4,65 \%$ & 0,0240 & $92,28 \%$ \\
\hline
\end{tabular}

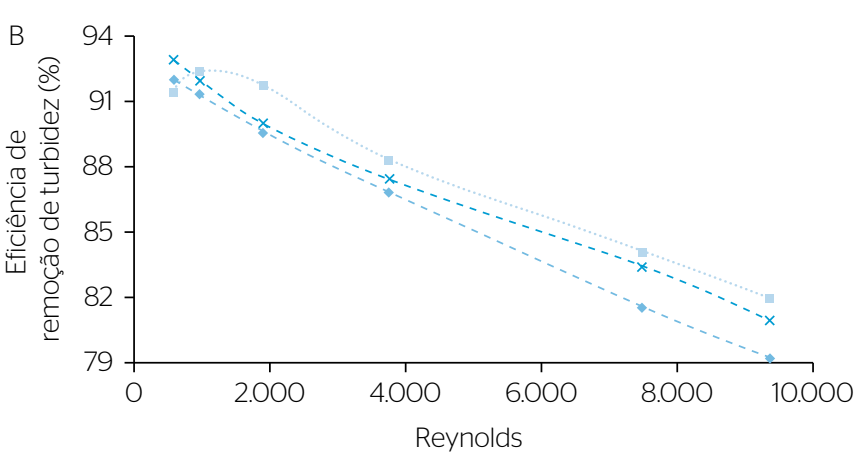

Tempo de processo adimensionalizado, relativo ao FTH de maior comprimento

Experimental $\rightarrow-$ - Oliveira (2014) $--\star--$ Proposta 
Para o caso ilustrado na Figura 6A, verificou-se que a formulação proposta captou melhor o comportamento ascendente-descendente da eficiência de remoção de turbidez ao longo do tempo de processo. Destaca-se que Oliveira (2014) verificou, para esse mesmo arranjo de FTHs ilustrado na Figura 6A, que a formulação de Argaman e Kaufman (1970) não conseguiu prever esse comportamento ascendente-descendente, de modo que foi observado um aumento da eficiência de forma assintótica com o tempo de processo.

Isso corre pois, segundo Marques e Ferreira Filho (2017), o modelo de Argaman e Kaufman (1970) considera que os processos de agregação e ruptura dos flocos ocorrem de forma simultânea e alcançam um estado de equilíbrio que permanece ao longo do tempo, desconsiderando o processo de ruptura irreversível dos flocos. Porém, na prática, o equilíbrio entre os dois processos pode ser apenas temporário, de modo a existir uma tendência de aumento da turbidez residual após determinado tempo de floculação. Libânio (1995) destaca que durante o processo de floculação os flocos crescem até atingir seu tamanho máximo. A partir desse instante, ocorre a predominância do mecanismo de ruptura, de maneira que à medida que se aumenta o tempo de floculação, tem-se a redução do tamanho dos flocos e da eficiência da floculação.

$\mathrm{Na}$ Figura 6B é ilustrada a maior capacidade da formulação proposta em prever os valores da eficiência de remoção de turbidez para escoamentos com número de Reynolds mais elevado. Enquanto o perfil produzido pela formulação de Oliveira (2014) apresenta tendência de se afastar dos dados experimentais. Com o aumento do número de
Reynolds do escoamento, o perfil gerado pela formulação proposta (Equação 14) se aproxima dos dados experimentais.

\section{CONCLUSÃO}

O desenvolvimento deste trabalho levou ao aperfeiçoamento do modelo proposto por Oliveira (2014), considerando os seguintes aspectos: a introdução do parâmetro $\mathrm{GP}_{\text {adm}}$, como variável independente, produziu formulação ligeiramente mais aderente ao processo físico, quando comparada à versão original, captando melhor as variações da eficiência de remoção de turbidez, em especial seu comportamento ascendente-descendente ao longo do tempo de processo, e o seu comportamento ao longo da escala do número de Reynolds (particularmente para valores mais elevados); a nova versão proposta tem um número menor de varáveis independentes, visto que os parâmetros helicidade adimensional $\left(\mathrm{H}_{\text {adm }}\right)$ e $\mathrm{Sn}$ foram eliminados da versão original por apresentarem baixo grau de significância; o modelo proposto tornou-se mais simples do ponto de vista operacional, pois o parâmetro Sn demandava grande esforço para sua aquisição via modelagem fluidodinâmica computacional.

\section{AGRADECIMENTO}

Os autores agradecem a professora Danieli Soares de Oliveira (Instituto Federal de Educação, Ciência e Tecnologia do Espírito Santo - IFES) pela disponibilização dos dados experimentais utilizados e pelo apoio na realização desta pesquisa.

\section{REFERÊNCIAS}

AL HASHIMI, M.A.I.; ASHJYAN, A.S. (1989) Effectiveness of helical pipes in the flocculation process of water. Filtration and Separation, v. 26, n. 6, p. $422-429$

ARGAMAN, Y.; KAUFMAN, W.J. (1970) Turbulence and flocculation. Journal of the Sanitary Engineering Division, v. 96, n. 2, p. 223-241.

BRATBY, J.; MILLER, M.W. (1977) Design of flocculation systems from batch test data. Water South Africa, v. 3, n. 4, p. 173-182.

BRIDGEMAN, J.; JEFFERSON, B.; PARSONS, S.A. (2009) Computational fluid dynamics modelling of flocculation in water treatment: a Review. Engineering Applications of Computational Fluid Mechanics, v. 3, n. 2, p. 220-241. https://doi.org/10.1080/1994 2060.2009.11015267

CARISSIMI, E.; RUBIO, J. (2005) The flocs generator reactor-FGR: a new basis for flocculation and solid-liquid separation. International Journal of Mineral Processing, v. 75, n. 3-4, p. 237-247. https://doi. org/10.1016/j.minpro.2004.08.021
CHIGIER, N.A.; BEER, J.M. (1964) Velocity and Static Pressure Distributions in Swirling Air Jets Issuing from Annular and Divergent Nozzles. Journal of Basic Engineering Transactions of the ASME, v. 86, n. 4, p. 788-796. https://doi. org/10.1115/1.3655954

CIONCOLINI, A.; SANTINI, L. (2006) An experimental investigation regarding the laminar to turbulent flow transition in helically coiled pipes. Experimental Thermal and Fluid Science, v. 30, n. 4, p. 367-380. https://doi.org/10.1016/j. expthermflusci.2005.08.005

GHOBADI, M.; MUZYCHKA, Y. (2016) A Review of Heat Transfer and Pressure Drop Correlations for Laminar Flow in Curved Circular Ducts. Heat Transfer Engineering, v. 37, n. 10, p. 815-839. https://doi. org/10.1080/01457632.2015.1089735

GROHMANN, A.; REITER, M.; WIESMANN, U. (1981) New flocculation units with high efficiency. Water Science Technology, v. 13, n. 11-12, p. 567-573. 
ISHAK, M.S.A.; JAAFAR, M.N.M. (2004) The effect of swirl number on discharge coefficient for various orifice sizes in a burner system. Journal Mekanikal, v. 17, p. 99-108.

KRAMER, T.A.; CLARK, M.M. (1997) Influence of strain-rate on coagulation kinetics. Journal of Environmental Engineering, v. 123, n. 5, p. 444-452. https://doi.org/10.1061/(ASCE)07339372(1997)123:5(444)

KÜHNEN, J.; BRAUNSHIER, P.; SCHWEGEL, M.; KUHLMANN, H. C.; HOF, B. (2O15) Subcritical versus supercritical transition to turbulence in curved pipes. Journal of Fluid Mechanics, v. 770, p. R3.1-R3.12. https://doi.org/10.1017/jfm.2015.184

LIBÂNIO, M. (1995) Avaliação da floculação em reatores estáticos e de escoamento contínuo com gradientes de velocidade constante e variável. 136f. Tese (Doutorado) - Escola de Engenharia de São Carlos, Universidade de São Paulo, São Carlos.

MARQUES, R.O.; FERREIRA FILHO, S.S. (2017) Flocculation kinetics of low-turbidity raw water and the irreversible floc breakup process. Environmental Technology, v. 38, n. 7. p. 901-910. https:// doi.org/10.1080/09593330.2016.1236149

MOFFATT, H.K.; TSINOBER, A. (1992) Helicity in laminar and turbulent flow. Annual Review of Fluid Mechanics, v. 24, n. 1, p. 281-312. https://doi.org/10.1146/annurev.fl.24.010192.001433

MORUZZI, R.B.; OLIVEIRA, S.C. (2O13) Mathematical modeling and analysis of the flocculation process in chambers in series. Bioprocess and Biosystems Engineering, v. 36, n. 3, p. 357-363. https://doi.org/10.1007/s00449-012-0791-4

OLIVEIRA, D.S. (2008) Avaliação da eficiência de remoção de turbidez em função de variações no comprimento de floculadores tubulares helicoidais. Dissertação (Mestrado em Engenharia Ambiental) - Programa de Pós-Graduação em Engenharia Ambiental, Universidade Federal do Espírito Santo, Vitória.
OLIVEIRA, D.S. (2014) Proposição de modelo de previsão de desempenho de unidades de floculação tubulares helicoidais. Tese (Doutorado em Engenharia Ambiental) - Programa de Pós-Graduação em Engenharia Ambiental, Universidade Federal do Espírito Santo, Vitória.

SARTORI, M.; OLIVEIRA, D.S.; TEIXEIRA, E.C.; RAUEN, W.B.; REIS JR., N.C. (2015) CFD modelling of helically coiled tube flocculators for velocity gradient assessment. Journal of the Brazilian Society of Mechanical Sciences and Engineering, v. 37, n. 1, p. 187-198. http:/l dx.doi.org/10.1007/s40430-014-0141-3

SILVA, R.C.D. (2007) Avaliação da Influência da geometria e Dinâmica do escoamento de Floculadores Tubulares Helicoidais na Redução de Turbidez, utilizando Modelagem Física. Dissertação (Mestrado em Engenharia Ambiental) - Programa de Pós-Graduação em Engenharia Ambiental, Universidade Federal do Espírito Santo, Vitória.

THOMAS, D.N.; JUDD, S.J.; FAWCETT, N. (1999) Flocculation modeling: a review. Water Research, v. 33, n. 7. p. 1579-1592. https:// doi.org/10.1016/S0043-1354(98)00392-3

VANELI, B.P. (2016) Aperfeiçoamento do modelo de estimativa da eficiência de remoção de turbidez em floculadores tubulares helicoidais: Compreensão da física do processo e sua inter-relação coma eficiência de tratamento. Dissertação(Mestrado emEngenharia Ambiental) - Programa de Pós-Graduação em Engenharia Ambiental, Universidade Federal do Espírito Santo, Vitória.

WEBSTER, D.R.; HUMPHREY, J.A.C. (1993) Experimental Observations of Flow Instability in a Helical Coil. Journal of Fluids Engineering, v. 115, n. 3, p. 436-443. https://doi.org/10.1115/1.2910157

YU, B.; ZHENG, B.; LIN, C.X.; PEÑA, O.J.; EBADIAN, M.A. (2003) Laser Doppler anemometry measurements of laminar flow in helical pipes. Experimental Thermal and Fluid Science, v. 27, n. 8, p. 855-865. http://dx.doi.org/10.1016/S0894-1777(O3)00058-X 